\title{
Simplified Invagination Pancreatojejunostomy During Pancreatoduoden- ectomy Which can be Safe in the Training of Young Surgeons
}

Kazuaki Shibuya $^{1,2}$, Takahiro Einama ${ }^{1,2}{ }^{*}$, Hironori Abe ${ }^{1}$, Ryo Kanazawa ${ }^{1}$, Takashi Suzuki ${ }^{1,2}$, Fumihiko Matsuzawa ${ }^{1,2}$, Khor Lee Wee ${ }^{1}$, Akinobu Taketomi ${ }^{2}$ and Kyuno Kenichi ${ }^{1}$

${ }^{1}$ Department of Surgery, ObihiroKyokai Hospital East 5 South 9, Obihiro, Hokkaido 080-0805, Japan

${ }^{2}$ Department of Gastroenterological Surgery, Graduate School of medicine, Hokkaido University, Kita 15, Nishi 7, Kita-ku, Sapporo 060-8638, Japan

\section{Abstract}

Publication History:

Received: August 24, 2016

Background: Invagination is one of the useful reconstruction method for pancreatic enteric anastomosis Accepted: October 11, 2016 during pancreatoduodenectomy (PD), but it is unclear what is the best. On the other hand, a safe and Published: October 13, 2016 easy procedure is needed for the stable outcomes and the safe training for young surgeon. The aim of this study was to evaluate our simplified invagination pancreaticojejunostomy.

\section{Keywords:}

Methods: This is a pilot study about a modified technique. Between January 2012 and March 2015, Invagination method,

22 consecutive cases of pancreatoduodenectomy performed with invagination pancreaticojejunostomy Pancreatoduodenectomy, Pancreatic were eligible for inclusion. We retrospectively analyzed the operative courses. fistula

Results: The median surgical time was 496 minutes (range 232-755). The median blood loss was $600 \mathrm{ml}$ (range 75-4335). The soft pancreas was nine patients (40.9\%). Only one patient developed postoperative pancreatic fistula (Grade B) (4.5\%). The median follow-up period was 13 months (range 2-29), and there were no cases of occlusion at the anastomotic site. When comparing inexperienced and skilled surgeons, no statistical significance was observed in the clinical and operative parameters.

Conclusion: Our invagination PJ can be an easy and simple method which carries a low incidence of POPF, and safe for training of young surgeons.

\section{Introduction}

Pancreatoduodenectomy (PD) is a surgical procedure performed for patients with malignant or benign diseases of the pancreatic head and periampullary region. There construction following PD varies among facilities and surgeons. Among reconstructions of PD, pancreatic-enteric anastomosis is the most important reconstruction because it can be a direct cause of postoperative pancreatic fistula (POPF), a serious complication that can lead to critical events such as bleeding, infection or sepsis. For the pancreatic-enteric anastomosis, invagination method and duct-to-duct anastomosis have been popularly used in many surgeons. Clinical trials have been published to clarify which is the best reconstruction method for pancreaticenteric anastomosis, but it is still debated $[1,2]$. We also have to train up young surgeons on the safety operation with less complication. To achieve both the training and the operative safety, a simple and safe method is needed.

In the present study, we introduce our simplified invagination pancreatojejunostomy (PJ) and evaluated the clinical course of $\mathrm{PD}$ patients in our institute.

\section{Methods}

\section{Study design and patients}

This is a pilot study about a modified technique. This study was a single center retrospective study. In our institution, we begun to adopt invagination PJ for the reconstruction of pancreatic-enteric anastomosis in 2012. Between January 2012 and March 2015, all PD were performed using invagination PJ and 22 consecutive cases were collected. The study was approved by the medical ethics committee of ObihiroKyokai Hospital.

\section{Definition of post operative complications}

Pancreatic fistula is defined as an amylase level detected in drained fluid at least triple the normal serum amylase levels that persist for more than 3 days. This definition is based upon guidelines from the International Study Group of Postoperative Pancreatic Fistula (ISGPF) [3]. The degree of pancreatic fistula is categorized into three grades based on the ISGPF guidelines: grade A (no clinical symptoms), grade B (infection treatable using conservative treatment) and grade $\mathrm{C}$ (intercurrent hemoperitoneum or septicemia requiring management in an Intensive Care Unit and surgical intervention). Postoperative complications were stratified according to the ClavienDindo classification.

\section{Statistical analysis}

Discrete data were presented as frequency or percentage and were analyzed for differences using Pearson's chi square test or Fisher's exact test, as appropriate. Continuous data are presented as medians and ranges, and variability was measured using standard deviation (SD). Continuous data were analyzed using an independent twosample t-test. All $\mathrm{P}$ values were two-sided, and a $\mathrm{P}$ value $<0.05$

"Corresponding Author: Dr. Takahiro Einama, Department of Gastroenterological Surgery I, Hokkaido University Graduate School of Medicine, Kita-ku, Kita 15, Nishi 7, Sapporo 060-8638, Tel: +81-155-22-6600, Japan; Email: titiuehahaue@hotmail.com

Citation: Shibuya K, Einama T, Abe H, Kanazawa R, Suzuki T, et al. (2017) Simplified Invagination Pancreatojejunostomy During Pancreatoduodenectomy Which can be Safe in the Training of Young Surgeons. Int J Surg Surgical Porced 2: 126. https://doi.org/10.15344/2456-4443/2017/126

Copyright: (c) 2017 Shibuya et al. This is an open-access article distributed under the terms of the Creative Commons Attribution License, which permits unrestricted use, distribution, and reproduction in any medium, provided the original author and source are credited. 
Citation: Shibuya K, Einama T, Abe H, Kanazawa R, Suzuki T, et al. (2017) Simplified Invagination Pancreatojejunostomy During Pancreatoduodenectomy Which can be Safe in the Training of Young Surgeons. Int J Surg Surgical Porced 2: 126. https://doi.org/10.15344/2456-4443/2017/126

Page 2 of 5

was considered statistically significant. All statistical analyses were performed using JMP Pro12 (SAS Institute Japan, Tokyo, Japan).

\section{Surgical technique}

After the specimen was resected, reconstructions are performed in the order of PJ, cholangiojejunostomy and gastrojejunostomy. Our invagination PJ involves two-layer interrupted sutures. The jejunum is opened to a length shorter than the width of the pancreas. Posterior outer sutures are placed through pancreatic serosa and jejunal seromuscular layer (Figure 1), starting at the superior margin of the pancreas and extending to the inferior margin. These sutures are placed approximately $4-5 \mathrm{~mm}$ from the edge of the pancreas, and are tied following placement. We must tie these sutures not to tear the pancreas. Posterior inner sutures are then placed through the pancreatic parenchyma and jejunal mucosa layer (Figure 2). The inner stitches are placed surrounding main pancreatic duct (Figure 3) with being careful not to suture the pancreatic duct. To keep the patency of main pancreatic duct, we place a stent. In brief, a simple two-layer anastomosis prevent the anastomotic leakage and a stent can keep the patency of main pancreatic duct. The stent is a complete external fistula to drainage pancreatic juice, and the opposite end of the stent is out from approximately $30 \mathrm{~cm}$ from PJ. Anterior inner and outer sutures are performed just like posterior sutures, invaginating the pancreas to the jejunum (Figure 3). Cholangiojejunostomy is one layer of continuous suture, and gastrojejunostomy is AlbertLembertanastomosis. When all reconstructions were finished, we make gastrostomy and place two drains in front and behind the PJ (Figure 4).

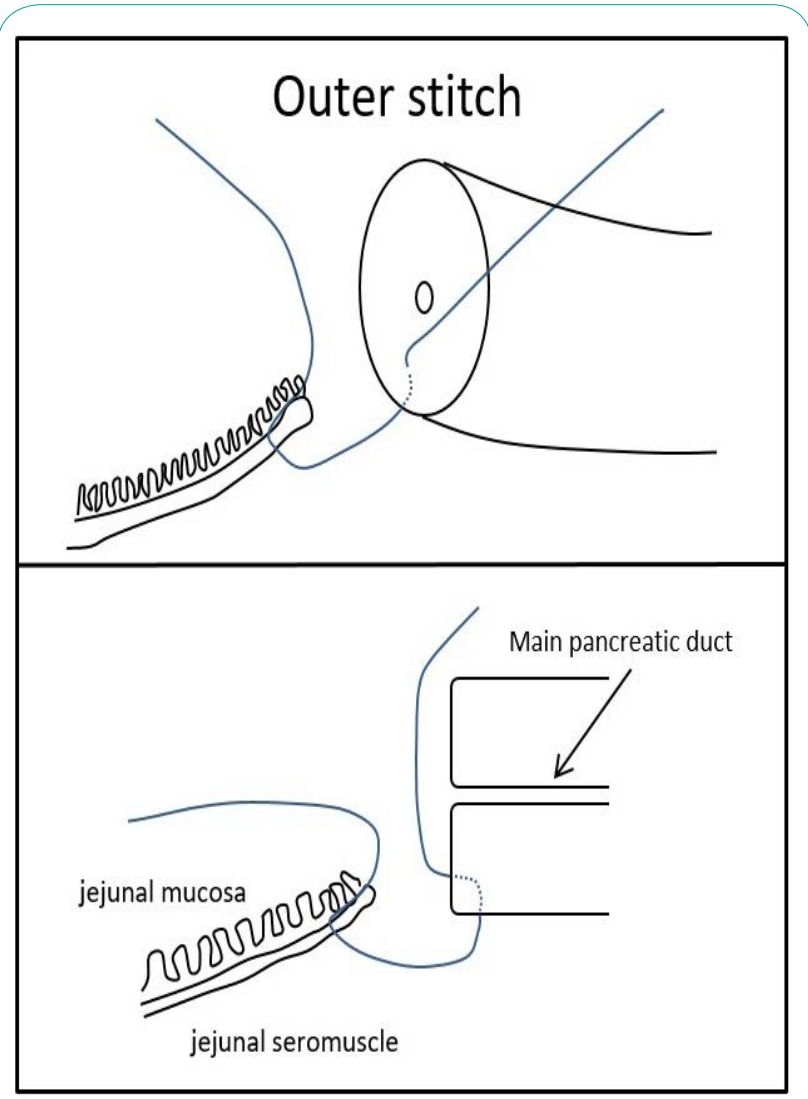

Figure 1: Posterior outer row of mattress sutures.

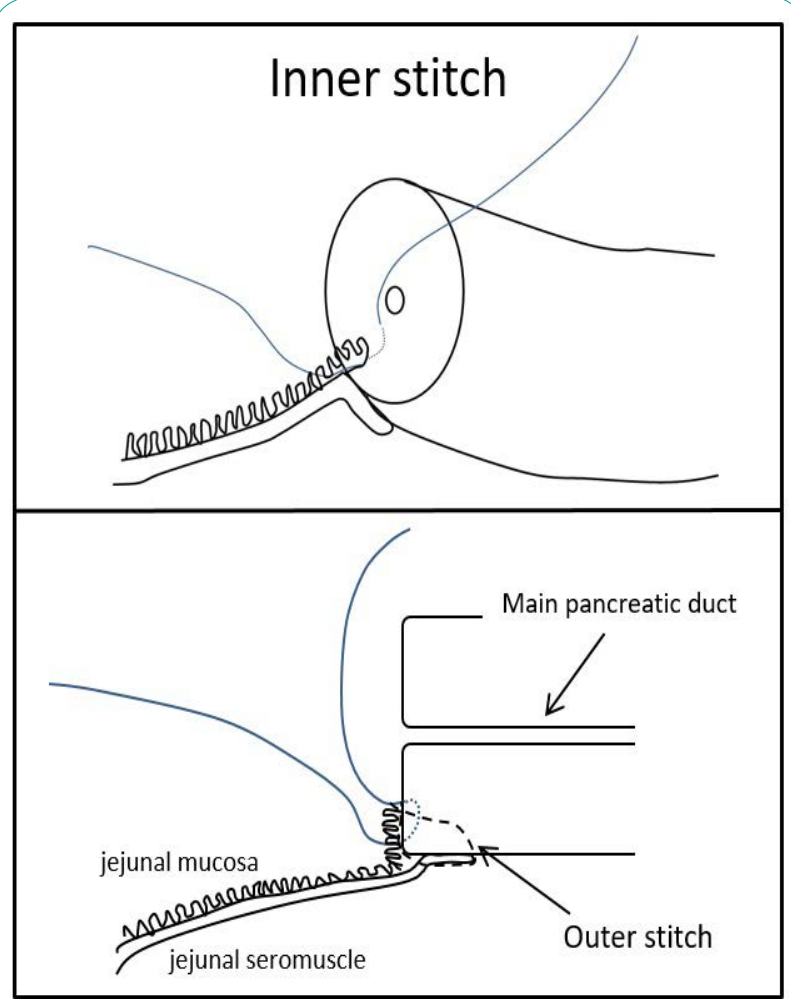

Figure 2: Posterior inner row of mattress sutures.

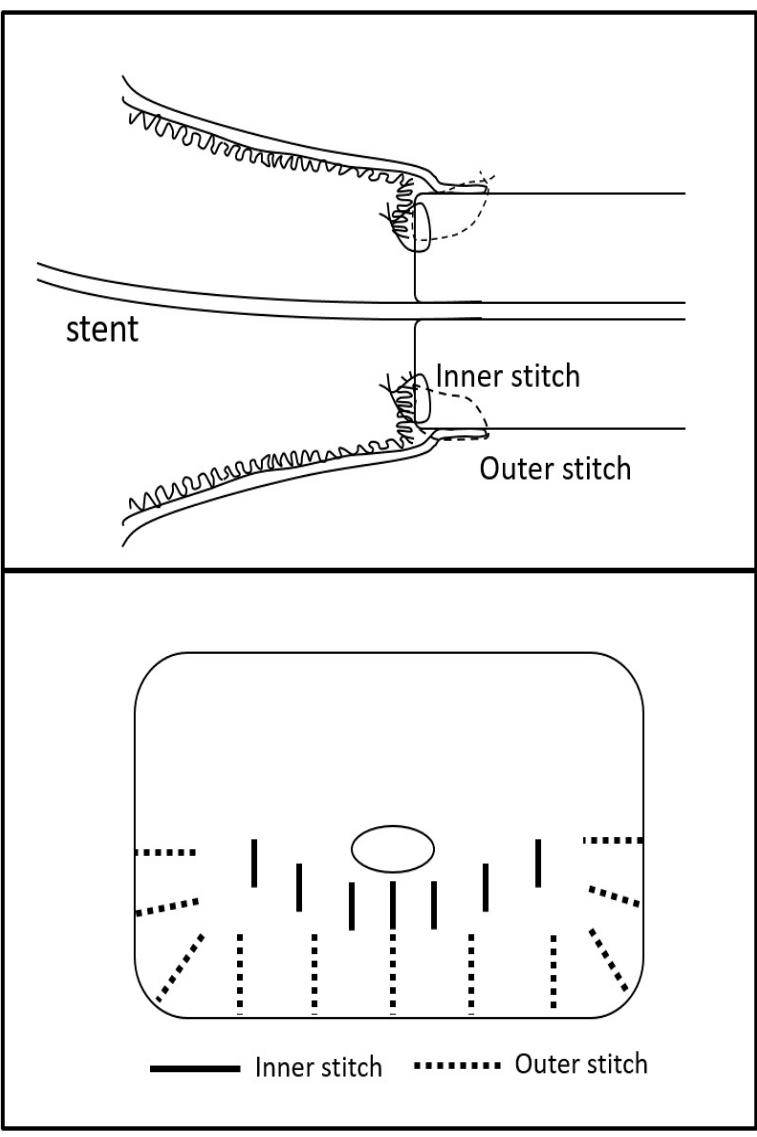

Figure 3: Section view of completed anastomosis. 
Citation: Shibuya K, Einama T, Abe H, Kanazawa R, Suzuki T, et al. (2017) Simplified Invagination Pancreatojejunostomy During Pancreatoduodenectomy Which can be Safe in the Training of Young Surgeons. Int J Surg Surgical Porced 2: 126. https://doi.org/10.15344/2456-4443/2017/126

Page 3 of 5

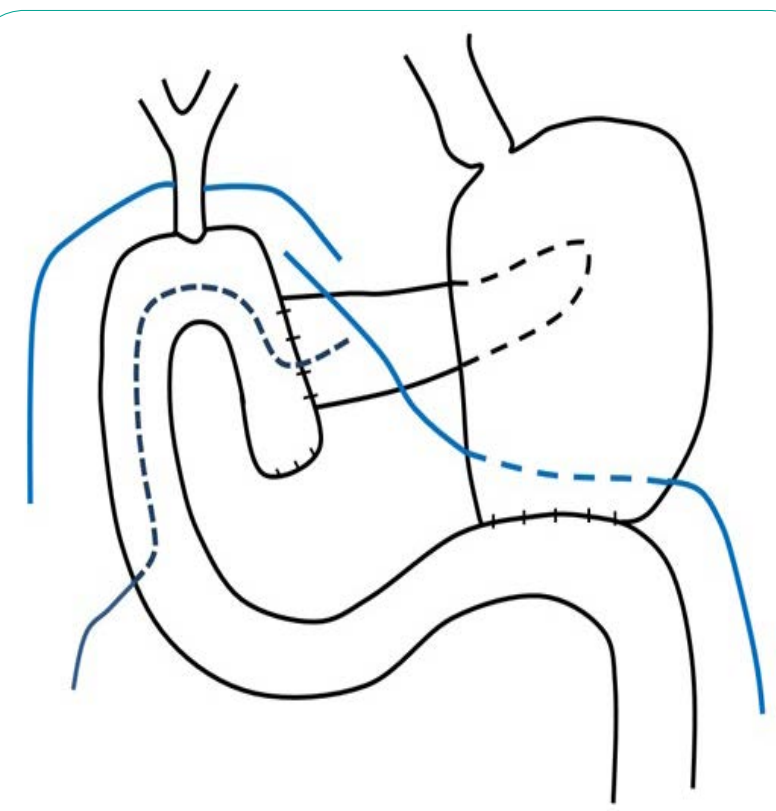

Figure 4: Finish of reconstructions.

\section{Results}

Patient characteristics are shown in (Table 1). This study involved 22 patients ( 12 females and 10 males). The median age was 67 (range 49-80) years. The pathological diagnoses included pancreatic ductal carcinoma in 12 patients, duodenal carcinoma in 4 patients, biliary ductal carcinoma in 3 patients and serous cystic adenoma in 3 patients. Our invagination PJ was used in all patients. The median surgical time was 496 minutes (range 232-755). The median blood loss was $600 \mathrm{~mL}$ range $75-4335$ ). The condition of the pancreas was soft in 9 patients (40.9\%) and hard in 13 patients (59.1\%). Median amylase level in the drained fluid on the third postoperative day was $42 \mathrm{IU} / \mathrm{mL}$ (range 7-1066). Three patients developed postoperative pancreatic fistulae (13.5\%), and among these there were 2 grade A cases $(9.0 \%)$ and 1 grade B case $(4.5 \%)$. No grade C cases were observed. The remaining 19 patients $(86.5 \%)$ did not develop POPF. Another complications occurred in 8 cases: 1 case of leakage from the cholangiojejunostomy (grade IIIa), 2 cases of gastric emptying (grade II), 2 cases of ileus (grade II) and 1 case of chylous ascites (grade I). Oral intake usually commenced on day 3 (range 3-16 days), and the median postoperative hospital stay was 25 days (range 14-96). The median follow-up period was 12 months (range 2-29), and there were no cases of occlusion at the anastomotic site with CT examination.

\begin{tabular}{|c|c|c|c|c|c|}
\hline & & All & $\begin{array}{c}\text { Inexperience } \\
\text { surgeon }\end{array}$ & Skilled surgeon & $\mathrm{p}$ \\
\hline \multicolumn{2}{|l|}{$\mathrm{n}$} & 22 & 4 & 18 & \\
\hline Age(years), Median(range) & & $67(49-80)$ & $68(67-74)$ & $66(49-80)$ & 0.46 \\
\hline \multicolumn{6}{|l|}{ Gender } \\
\hline & \begin{tabular}{|l|} 
Male \\
Female
\end{tabular} & $\begin{array}{l}10 \\
10 \\
\end{array}$ & $\begin{array}{l}2 \\
2\end{array}$ & $\begin{array}{c}7 \\
11 \\
\end{array}$ & 1 \\
\hline \multicolumn{6}{|l|}{ Pancreas Condition } \\
\hline & $\begin{array}{l}\text { Soft } \\
\text { Hard }\end{array}$ & $\begin{array}{c}9 \\
13 \\
\end{array}$ & $\begin{array}{l}3 \\
1\end{array}$ & $\begin{array}{c}6 \\
12 \\
\end{array}$ & 0.26 \\
\hline \multicolumn{6}{|l|}{ Pathological Diagnosis } \\
\hline & Pancreatic duct carcinoma & 12 & 1 & 11 & \\
\hline & Duodenum carcinoma & 4 & 1 & 3 & \\
\hline & Bilialy ductal carcinoma & 3 & 0 & 2 & \\
\hline & Serous cysitic adenoma & 3 & 2 & 1 & \\
\hline & $\begin{array}{l}\text { Operation time (min), median } \\
\text { (range) }\end{array}$ & $496(232-755)$ & $507(232-687)$ & $496(307-755)$ & 0.93 \\
\hline & Blood loss (ml),median(range) & $600(75-4335)$ & $326.5(115-4335)$ & $640(75-2078)$ & 0.69 \\
\hline & $\begin{array}{l}\text { Amylase in drain (3POD) (IU/ } \\
\text { ml), median (range) }\end{array}$ & $42(7-1066)$ & $569(97-1066)$ & $26(7-334)$ & 0.09 \\
\hline \multicolumn{6}{|l|}{ The Grade of Pancreatic Fistula } \\
\hline & No grade & 19 & 2 & 17 & \\
\hline & Grade A & 2 & 1 & 1 & \\
\hline & Grade B & 1 & 1 & 0 & \\
\hline & Grade C & 0 & 0 & 0 & \\
\hline \multirow[t]{7}{*}{ Post Operative Complication } & & $8(36 \%)$ & & & \\
\hline & Clevien-Dindo & & & & \\
\hline & I & 1 & 0 & $1 * 1$ & 1 \\
\hline & II & 4 & 0 & $4^{\star} 2$ & 0.54 \\
\hline & IIIa & 1 & $1 * 3$ & 0 & 1 \\
\hline & $\mathrm{IVb}$ & 0 & 0 & 0 & \\
\hline & $\mathrm{V}$ & 0 & 0 & 0 & \\
\hline Post Operative hospitalization(day)(median) & & $25(14-96)$ & $23(21-42)$ & $27(14-96)$ & 0.8 \\
\hline
\end{tabular}


Citation: Shibuya K, Einama T, Abe H, Kanazawa R, Suzuki T, et al. (2017) Simplified Invagination Pancreatojejunostomy During Pancreatoduodenectomy Which can be Safe in the Training of Young Surgeons. Int J Surg Surgical Porced 2: 126. https://doi.org/10.15344/2456-4443/2017/126

Page 4 of 5

\begin{abstract}
Among the 22 cases, four cases were performed by inexperienced surgeons as their first experience of PD, whereas skilled surgeons performed PD for the remaining 18 cases. There were no significant differences between these groups in terms of age or gender. A soft pancreas was found more often in the inexperienced surgeon group with no significant difference $(\mathrm{p}=0.26)$. When comparing the inexperienced surgeon group with the skilled surgeon group, the median surgical time was 507 vs $496 \mathrm{~min}(\mathrm{p}=0.93)$, the median blood loss was 326.5 vs $640 \mathrm{~mL}(\mathrm{p}=0.69)$, the median amylase level in the drained fluid on postoperative day 3 was 596 vs $26 \mathrm{IU} / \mathrm{mL}(\mathrm{p}=0.09)$ and the postoperative hospital stay was 23 vs 27 days $(p=0.80)$ with no significant difference. Grade B POPF was occurred in the inexperienced surgeon group [ $\mathrm{p}=0.18]$. The median follow-up period was 13 months. No patients have had morphological change of the residual pancreas in CT, including dilation of main pancreatic duct or atrophy of pancreatic parenchyma, and have developed the abnormal glucose tolerance.
\end{abstract}

\section{Discussion}

Randomized controlled trials have been published to investigate the best reconstruction method for PD. These trials, which were carried out from high volume centers, have compared invagination to duct-to-mucosa anastomosis [4-9] or pancreatogastrostomy to pancreatojejunostomy [10-16]. However, we have not obtained a clear consensus as to which reconstruction method is the best for pancreatic-enteric anastomosis. POPF which can lead critical events is the primary endpoint to evaluate the pancreatic-enteric anastomosis. According to these reports, the POPF rates were $4 \%$ $18 \%$ in duct-to-mucosa anastomosis, $3 \%-15 \%$ in invagination, $8 \%$ $25 \%$ inpancreatogastrostomy and $11 \%-34 \%$ in pancreatojejunostomy [4-16]. Totally, the published papers concluded that invagination orpancreatogastrostomy can be a little better than duct-to-mucosa anastomosisor pancreatojejunostomy. Invagination method is simple and easy. In addition, PJ is more natural than pancreatogastrostomy which needs the extra mobilization of the residual pancreas. Hence, we choice PJ invagination as a pancreatic-enteric anastomosis for its simplicity. The rate of POPF incidence (Grade $B \leq$ ) of our method was $4.5 \%$ which may be lower compared with earlier studies.

Of course, these results cannot be simply compared because of the difference of the hospital scale, such as high-volume centers and medium-scale hospitals like our institution. In high-volume centers where many PD cases performed, surgeons and medical staffs will provide a more skillful care sophisticatedly, furthermore, surgeons may be able to try different reconstructions or select an appropriate reconstruction based on each case. In contrast to high volume centers, we performed at most 10 cases of PD per year. The most important point to prevent unexpected accidents and complications in a medium scale hospitals is to perform an accomplished procedure routinely. The routine skill can provide certainty and results in the sense of security for operator and staffs during the operation. Moreover, considering that one of roles in our department is the training of young surgeons, the development of a simple and safe reconstruction method which is appreciated for inexperienced surgeons facing their first experience of $\mathrm{PD}$ is necessary.

As an identified operative method, our invagination $\mathrm{PJ}$ protocol is simple which carries a low POPF. The concept of this method is drainage of pancreatic juice into the jejunum. POPF is occurred not only from the main pancreatic duct but also the branches or the capsule defect of the residual pancreas. In our method, the edge of the residual pancreas is invaginated into the jejunum, and the pancreatic juice from the main pancreatic duct and also its branches or parenchyma runs through the jejunum. The advantage of this method is unnecessity of suturing the pancreatic duct, and no matter how narrow the pancreatic duct is, we can perform the anastomosis with less stress. On the other hand, the problem of the invagination method is a low patency rate in the long term perspective because of no suturing of the main pancreatic duct, but it is the most advantage in invagination PJ. In our institution, occlusion of anastomosis orpancreatic morphological change have not been observed, neither has the abnormal glucose tolerance. Otherwise, the advantage of ductto-mucosa anastomosis is a long term good patency rate due to the complete drainage of pancreatic main duct to suture the pancreatic mucosa and jejunal mucosa. But this technique requires a detailed work. The smaller the pancreatic duct is, the more difficult the anastomosis may be. Invagination PJ is easier than duct-to-mucosa anastomosis, especially in a narrow pancreatic duct.

Research demonstrated the relation between POPF and the skills of surgeons is less. Tobias et al. reported no statistical association between POPF and surgeon's experience [16]. However, surgeons defined in the report were specialized in the pancreas. The inexperienced surgeons defined in our study are 2-3 years post residency and not a specialized pancreatic surgeon. Showing [Table 1], no significant differences were observed in patient characteristics and operative factors between inexperienced and skilled surgeons. However, the incidence of soft pancreas, which is a risk factor for POPF $[14,15]$, was higher in inexperienced arm despite no significant difference. Considering the high incidence of soft pancreas in the inexperienced arm, we think our POPF rate in the inexperienced arm is not high. The case of grade B POPF was a man and the soft pancreas, both of which are risk factors for POPF $[8,14,15]$.

Our study has some limitations. This study was a retrospective study, the sample size was small, and the follow-up duration was relatively short. More cases and the long-term data should be necessary.

In conclusion, our invagination PJ can be an easy and simple method which carries a low incidence of POPF, and safe for training of young surgeons.

\section{Conflict of interest}

No authors have a conflict of interest or any financial tie to disclose.

\section{References}

1. Crippa S, Cirocchi R, Randolph J, Partelli S, Belfiori G, et al. (2016) Pancreaticojejunostomy is comparable to pancreaticogastrostomy after pancreaticoduodenectomy: an updated meta-analysis of randomized controlled trials. Langenbecks Arch Surg 401: 427-437

2. Lei P, Fang J, Huang Y, Zheng Z, Wei B, et al. (2014) Pancreaticogastrostomy or pancreaticojejunostomy? Methods of digestive continuity reconstruction after pancreaticodudenectomy: A meta-analysis of randomized controlled trials. Int J Surg 12: 1444-1449.

3. Bassi C, Dervenis C, Butturini G, Fingerhut A, Yeo C, et al. (2005) Postoperative pancreatic fistula: an international study group (ISGPF) definition. Surgery 138: 8-13.

4. Bassi C, Falconi M, Molinari E, Mantovani W, Butturini G, et al. (2003) Duct-to-mucosa versus end-to-side pancreaticojejunostomy reconstruction after pancreaticoduodenectomy: results of a prospective randomized trial. Surgery 134: 766-771. 
Citation: Shibuya K, Einama T, Abe H, Kanazawa R, Suzuki T, et al. (2017) Simplified Invagination Pancreatojejunostomy During Pancreatoduodenectomy Which can be Safe in the Training of Young Surgeons. Int J Surg Surgical Porced 2: 126. https://doi.org/10.15344/2456-4443/2017/126

5. Berger AC, Howard TJ, Kennedy EP, Sauter PK, Bower-Cherry M, et al. (2009)Does type of pancreaticojejunostomy after pancreaticoduodenectomy decrease rate of pancreatic fistula? A randomized, prospective, dualinstitution trial. J Am Coll Surg 208: 738-747.

6. Langrehr JM, Bahra M, Jacob D, Glanemann M, Neuhaus P (2005) Prospective randomized comparison between a new mattress technique and Cattell (duct-to-mucosa) pancreaticojejunostomy for pancreatic resection. World J Surg 29: 1111-1119

7. Xu J, Zhang B, Shi S, Qin Y, Ji S, et al. (2015) Papillary-like main pancreatic duct invaginated pancreaticojejunostomy versus duct-to-mucosa pancreaticojejunostomy after pancreaticoduodenectomy: A prospective randomized trial. Surgery 158: 1211-1218.

8. Bai X, Zhang Q, Gao S, Lou J, Li G, et al. (2016) Duct-to-Mucosa vs Invagination for Pancreaticojejunostomy after Pancreaticoduodenectomy: A Prospective, Randomized Controlled Trial from a Single Surgeon. Journa of the American College of Surgeons 222: 10-18.

9. Hua J, He Z, Qian D, Meng H, Zhou B, et al. (2015) Duct-to-Mucosa Versus Invagination Pancreaticojejunostomy Following Pancreaticoduodenectomy: a Systematic Review and Meta-Analysis. J Gastrointest Surg 19: 19001909.

10. Wellner U, Makowiec F, Fischer E, Hopt UT, Keck T (2009) Reduced postoperative pancreatic fistula rate after pancreatogastrostomy versus pancreaticojejunostomy. J Gastrointest Surg 13: 745-751.

11. Yeo CJ, Cameron JL, Maher MM, Sauter PK, Zahurak ML, et al. (1995) A prospective randomized trial of pancreaticogastrostomy versus pancreaticojejunostomy after pancreaticoduodenectomy. Ann Surg 222 580-588.

12. Bassi C, Falconi M, Molinari E, Salvia R, Butturini G, et al. (2005) Reconstruction by pancreaticojejunostomy versus pancreaticogastrostomy following pancreatectomy: results of a comparative study. Ann Surg 242: 767-771.

13. Topal B, Fieuws S, Aerts R, Weerts J, Feryn T, et al. (2013) Pancreaticojejunostomy versus pancreaticogastrostomy reconstruction after pancreaticoduodenectomy for pancreatic or periampullary tumours: a multicentrerandomised trial. Lancet Oncol 14: 655-662.

14. Figueras J, Sabater L, Planellas P, Munoz-Forner E, Lopez-Ben S, et al. (2013) Randomized clinical trial of pancreaticogastrostomy versus pancreaticojejunostomy on the rate and severity of pancreatic fistula after pancreaticoduodenectomy. Br J Surg 100: 1597-1605.

15. Grendar J, Ouellet JF, Sutherland FR, Bathe OF, Ball CG, et al. (2015) In search of the best reconstructive technique after pancreaticoduodenectomy: pancreaticojejunostomy versus pancreaticogastrostomy. Can J Surg 58: 154-159.

16. Keck T, Wellner UF, Bahra M, Klein F, Sick O, et al. (2016) Pancreatogastrostomy Versus Pancreatojejunostomy for Reconstruction After Pancreatoduodenectomy (RECOPANC, DRKS 00000767): Perioperative and Long-term Results of a Multicenter Randomized Controlled Trial. Ann Surg 263: 440-449.

This article was originally published in a special issue: Upper Gastrointestinal Surgery

Handled by Editor(s):

Dr. CE Costa Almeida

Department of Surgery

Hospital and University Centre of Coimbra

University of Coimbra

Portugal 